\title{
Unended Reflections on Family Resemblance and Predicates Linguistic Migration*
}

\author{
I. García-Honrado ${ }^{\dagger}$ E. Trillas
}

European Centre for Soft Computing. Mieres (Asturias), Spain.

\begin{abstract}
This paper takes into account the Wittgenstein's idea of family resemblance as a particular crisp relation between some fuzzy sets, that is, between some representations of predicates use from its use. It is shown that all uses of the same predicate actually do have some kind of family resemblance, that some pairs of predicates cannot, and how the predicates migration between different universes of discourse can be related with family resemblance. A possibility of sketching all that through Galois Connections is also considered.
\end{abstract}

Keywords: Fuzzy sets, Family resemblance, Predicates linguistic migration.

\section{Introduction}

\section{1 .}

Ludwig Wittgenstein headed two influential traditions in the so-called philosophy of language, that were originated by his famous books, Tractatus logico-philosophicus (1922, [13]), and Philosophical Investigations (1953, [12]), respectively. The Tractatus deals with the logical analysis of propositions, considered as 'pictures' of facts and keeping a strict correspondence with the facts in the world.

Is in the Philosophical Investigations where Wittgenstein abandoned logical analysis by conferring a main role to the ways of designating facts, as a dynamic activity-oriented perspective on language. What is central at this respect, is that language consists on playing 'language games', or ways of dynamically using words to establish their meaning. In order to fix the meaning of a word, to show how a word works, it should be placed in the context and environment it is used, and to note the absence of sharp boundaries for describing such use of words, Wittgenstein introduced the term 'family resemblance'. Of course, Wittgenstein's idea on 'family resemblance', is broader than the relations of family resemblance and migration here considered between fuzzy sets.

${ }^{*}$ This work has been partially supported by the Foundation for the Advancement of Soft Computing (Asturias, Spain), and CICYT (Spain) under project TIN2008-06890C02-01

${ }^{\dagger}$ Email: itziar.garcia@softcomputing.es
Fuzzy logic manages the extensional meaning of predicates through its use, once captured by the corresponding membership functions. This paper is nothing else than a first approach, in the path towards Zadeh's Computing With Words, to introduce 'family resemblance' between fuzzy sets.

In its own nature, it is not a conclusive paper, but only a tentative to reflect on the potentially interesting subject of the family resemblances shown by fuzzy sets. That is, 'the development over time of what can be predicated of the sets of objects falling under some concept' [6].

\section{2 .}

The question behind this paper is how to obtain a first mathematical model for the linguistic phenomenon (part of what Ludwig Wittgenstein called 'language games') under which words successively acquire diverse meanings in language, and how are they related. This phenomenon, related with Wittgenstein's family resemblance, could be designated as the linguistic migration of predicates.

Remark 1.1 Antecedents of what has been said are the following

- People in a family can be similar respect to several physical characteristics: color of eyes or hair, way of walking, temperament, etc., but very different on what concerns other characteristics.

- Mountains are older than basketball teams. Hence, it can be supposed that the term, for instance, 'tall' migrated form designating 'tall mountains' to designate 'tall basketball players'.

- The word 'indian', initially denoting an inhabitant of India, migrated to denote American natives.

From a, let us call 'dynamic' perspective, there are some noticeable and interesting questions that can be posed through the following points.

1. A predicate $P$ is introduced in language to name some objects $x_{1}, \ldots, x_{n}$ and, over time, it acquires a certain meaning $\left(\leq_{P}, \mu_{P}\right)$, with $\mu_{P}: X \rightarrow L$ a degree, $X$ the final universe of discourse to which $P$ applies (including the initial objects $\left.x_{i}\right)$, and $\mathcal{L}=(L, \leq, 0,1)$ some 
partially ordered set with extremes 0,1 , suitable for assigning a degree to $P$ (see sections 3 and 4).

2. Because of some similarity between the objects in $X$ and those in a new universe of discourse $X^{*}$, the predicate $P$ (renamed $P^{*}$ ) passes to be applied to the objects $x^{*}$ in $X^{*}$, acquiring a meaning $\left(\leq_{P}^{*}, \mu_{P}^{*}\right)$, where $\mu_{P}^{*}: X^{*} \rightarrow L^{*}$, and $\mathcal{L}^{*}=\left(L^{*}, \leq^{*}, 0^{*}, 1^{*}\right)$ is a partially ordered set. At this point, the relationship between the respective prototypes in $X$ and $X^{*}$ is relevant. Then, for instance, the following questions arise in the form,

(a) Which kind of algebraic relationship could exist between $\mu_{P}$ and $\mu_{P}^{*}$ ?

(b) Under which conditions, if $\mu_{P}$ perfectly reflects $\leq_{P}$ also $\mu_{P}^{*}$ perfectly reflects $\leq_{P}^{*} ?$

(c) After some of these migrations of $\mathrm{P}$ to some universes of discourse, is there a final meaning acquired by $P$ ? If such meaning does exist, how can it be mathematically expressed? This is usually the first meaning appearing in dictionaries.

This paper tries to do some first (and unended) reflection on questions (a) and (b).

Along the paper, it is dealt with full-normalized fuzzy sets, $\mu_{P} \in[0,1]^{X}$, those for which there are $x, y \in X$ such that $\mu_{P}(x)=1, \mu_{P}(y)=0$, that appear as 'data' in the modeling of fuzzy systems. Points $x$ verifying $\mu_{P}(x)=1$ can be taken as the prototypes of $P$ in $X$, and points $y$ verifying $\mu_{P}(y)=0$ as the anti-prototypes of $P$ in $X$.

\section{Basic Concepts}

Correctly managing a language at least implies to know what its expressions mean, that is, how to properly use them at each situation. Following Ludwig Wittgenstein [12], 'the meaning of a word is its use in the language' and, of course, the first problem for capturing the meaning of a predicate lies in how to describe and represent its use. Without 'representation' there is not the possibility of establishing a useful 'Computing with Words' like that advocated by Zadeh ([15]).

A predicate $P$ means nothing by itself, it only can mean something when acting on some universe of objects, $X$, the universe of discourse, of which it will be here supposed is a set. Such action is made though the elemental statements ' $x$ is $P$ ', and to know this action implies to know, at least, some of the basic rules by which the statements ' $x$ is $P$ ', ' $y$ is $P^{\prime}, \ldots$ are related. This paper only deals with those predicates reflecting 'collective nouns', that is, generating collectives in the universe they act in, or work, or are used. Actually, collectives are in the language and in the same form in which, for instance, in a big population, $P=$ 'short' allows to talk of its short inhabitants.

Once some elemental treats of the use of $P$ on $X$ are described, a way for representing the meaning of $P$ in $X$ can be introduced and the general concept of the degree up to which $x$ is $P$, can be tentatively defined. This is done in a form making clear that several value places, or scales, for the degrees are possible. It should be noticed that predicates appearing in the language were usually introduced by naming a property exhibited by some elements in a first 'universe of discourse'. After this, it is frequently the case that the considered predicate migrates to another universe of discourse, and that its use results in some form distorted, but showing some 'family resemblance' with its former use.

Most of the predicates originating collectives are such that these collectives, by lacking sharp boundaries, are not sets. This reflects what is commonly stated as 'the greys' their uses show, that is, the gradation under which the elements in the universe of discourse do verify the property named by the predicate. In this sense, the intuitive idea the word 'collective' tries to express is not representable with the same kind of uniqueness than that expressed by the word 'set', although evidently 'sets' are particular cases of 'collectives'. The word 'collective' reflects an abstract concept, and the corresponding representations are formal, mathematical, precisiations of it.

Words are for describing some reality, be it perceived in the real, or in an intellectual world, be it invented in a fictitious one, be it in a static or dynamic way. Those realities are seldom isolated ones, but placed in a context that can produce restrictions in the word use, as it happens with the work 'old' when used in different human contexts. Hence, the meaning or use of the words depends on the corresponding purpose for which they are used in a given context and evolve over time [6]. This is a relevant part of how words work.

\section{Primary or elemental meaning of $P$ in $X$}

If someone states 'I do manage $P$ on $X$ ', she/he should at least recognize when ' $y$ is less $P$ than $x$ ' or when ' $x$ is more $P$ than $y$ ', similarly to how in the Montessori's learning method [1], children learn the concept of length by ordering several sticks of different sizes to arrive at the idea of having the same length. In this sense, $P$ appears as a kind of quantity.

By taking the (empirical) relation $x$ is less 
$P$ than $y$, the corresponding algebraic relation $\leq_{P} \subset X \times X$, is obtained in $X$, and $y \leq_{P}^{-1} x(\Leftrightarrow$ $\left.x \leq_{P} y\right)$ can be identified with ' $y$ is more $P$ than $x$ '.

Considering the working hypotheses

1. $={ }_{P}=\leq_{P} \cap \leq_{P}^{-1}$ (translating $x$ is equally $P$ than $y$ ),

2 . $\leq_{P}$ is a preorder (a reflexive and transitive relation),

it is $={ }_{P}$ an equivalence relation giving the quotientset $X /{ }_{P}$, with classes $[x]=\left\{y \in X ; x={ }_{P} y\right\}=$ $\left\{y \in X ; x \leq_{P} y \& x \leq_{P}^{-1} y\right\}$. Under hypothesis (1), the only relation to be taken into account is $\leq_{P}$ that can be read as the organization made in $X$ by the use of $P$ in it. Of course, if $x=y$, it can be accepted that $x={ }_{P} y$, but not reciprocally in general.

Definition 3.1 $\leq_{P}$ is the primary, or elemental, meaning of $P$ in $X$, and $P$ is meaningless in $X$ if $\leq_{P}=\emptyset$.

Of course, if $P$ is meaningless in $X$, it is also $\leq_{P}^{-1}=\emptyset$ and $={ }_{P}=\emptyset$ : There is no way of 'organizing' the universe of discourse $X$.

\section{Remarks 3.2}

1. If $S=$ small, and $X=[0,10], S$ is usually acting on $X$ under the rule ' $x \leq_{S} y \Leftrightarrow y \leq x$ in the linear order of $\mathbb{R}$ '. Hence, $\leq_{S}^{-1}=\leq$, and ' $x={ }_{P} y \Leftrightarrow x=y$ '.

2. If $X$ is the set of inhabitants in a big city, and $P=$ short, it is usually considered that

$$
x \leq_{P} y \Leftrightarrow \text { Height of } x \leq \text { Height of } y,
$$

and $x={ }_{P} y \Leftrightarrow$ Height of $y=$ Height of $x$, once the heights are measured with a given accuracy. Obviously, $\leq_{P}$ is a preorder.

3. In $X=\mathbb{N}$, the predicate $P=$ 'transparent' is meaningless $\left(\leq_{P}=\emptyset\right)$, unless if it is possible to define ' $n$ is transparent' by either a necessary and sufficient condition, or describing a relation like ' $n$ is less transparent than $m$ '.

\section{The concept of degree}

\section{1 .}

Once the structure $\left(X, \leq_{P}\right)$ is established, an important question lies in how and where to measure up to which extent $x$ is $P$ (for all $x$ in $X$ ). Provided a partially ordered set $\mathfrak{L}=(L, \leq)$ can be, in some form, associated to $\left(X, \leq_{P}\right)$ for such purpose, a function $\mu_{P}: X \rightarrow L$ is an $\mathfrak{L}$-degree for $P$ in $X$, if

$$
x \leq_{P} y \Rightarrow \mu_{P}(x) \leq \mu_{P}(y) .
$$

Obviously, if $x={ }_{P} y$ it follows $\mu_{P}(x) \leq \mu_{P}(y)$ and $\mu_{P}(y) \leq \mu_{P}(x)$, that is, $\mu_{P}(x)=\mu_{P}(y)$ (see $[7],[9],[8],[10])$. The set $L^{X}$ can be viewed as a repository of potential degrees for predicates, and the poset $\mathfrak{L}$ as a scale for the degrees.

Theorem 4.1 The degree's concept is not an empty one.

Proof. Take the quotient set $X /=_{P}$, and translate $\leq_{P}$ to its classes by

$$
[x] \leq_{P}^{*}[y] \Leftrightarrow x \leq_{P} y .
$$

This definition does not depend on the chosen representatives of the classes and is a partial order. Hence, $\mathfrak{L}_{P}=\left(X /=_{P}, \leq_{P}^{*}\right)$ is a poset naturally linked to $\left(X, \leq_{P}\right)$.

Take any poset $(L, \leq)$ isomorphic to $\mathfrak{L}_{P}$, and define $\mu_{P}: X \rightarrow L$ by $\mu_{P}(x)=r$, with $r$ the element in $L$ that corresponds to the class $[x]$ by the isomorphism with $\left(X /=_{P}, \leq_{P}^{*}\right)$. Obviously,

$$
x \leq_{P} y \Leftrightarrow[x] \leq_{P}^{*}[y] \Rightarrow \mu_{P}(x) \leq \mu_{P}(y) .
$$

Hence, $\mu_{P}$ is an $L$-degree for $P$ in $X$.

For example, if $X=[0,6], P=$ close to four and $\mathcal{L}=([0,1], \leq)$, it can be stated

$x \leq_{P} y \Leftrightarrow x \leq y$, if $x, y \in[0,4]$ and $y \leq x$ if $x, y \in[4,6]$,

and any $L$-degree $\mu_{P}$ for $P=$ close to four, will be a non-decreasing numerical function between 0 and 4 , and decreasing between 4 and 6 .

Once an $L$-degree $\mu_{P}$ is defined in $\left(X, \leq_{P}\right)$, it can be considered the new relation $\leq_{\mu_{P}} \subset X \times X$, defined by

$$
x \leq_{\mu_{P}} y \Leftrightarrow \mu_{P}(x) \leq \mu_{P}(y) .
$$

Obviously: $x \leq_{P} y \Rightarrow x \leq_{\mu_{P}} y$, or $\leq_{P} \subset \leq_{\mu_{P}}$, that is, the relation $\leq_{\mu_{P}}$ is larger than the relation $\leq_{P}$. When $\leq_{P}=\leq_{\mu_{P}}$ it can be said that $\mu_{P}$ perfectly reflects the primary meaning, or use, of $P$ in $X$. When $\leq_{P} \varsubsetneqq \leq_{\mu_{P}}, \mu_{P}$ only reflects partially the primary use of $P$. The pair $\left(\leq_{P}, \leq_{\mu_{P}}\right)$ could be called the meaning, or use, of $P$ in $X$.

It should be noticed that the meaning, as defined in this way, is not of an absolute character but relative to the scale $\mathfrak{L}$ in which the degree $\mu_{P}$ takes its values.

\section{Remarks 4.2}

1. Obviously, $\leq_{\mu_{P}}$ is also a preorder, that is not always antisymmetric.

2. Zadeh's degrees do appear when $L=[0,1]$ and $\leq$ is the usual order of $\mathbb{R}$ [14]. In this case, what often is first established is a $[0,1]$-degree $\mu_{P}$, and hence, what is actually known is $\leq_{\mu_{P}}$, but not $\leq_{P}$. 
3. With $=_{\mu_{P}}=\leq_{\mu_{P}} \cap \leq_{\mu_{P}}^{-1}$, it results $x={ }_{\mu_{P}}$ $y \Leftrightarrow \mu_{P}(x)=\mu_{P}(y)$. Obviously, $x=y \Rightarrow$ $x={ }_{P} y \Rightarrow \mu_{P}(x)=\mu_{P}(y) \Leftrightarrow x=\mu_{P} y$. What is obtained is the chain of inclusions: $=\subset={ }_{P}$ $\subset=\mu_{P}$. Usually, this chain is strict.

4. Any constant function $\mu_{r}: X \rightarrow L, \mu_{r}(x)=$ $r \in L$ for all $x \in X$, 'can be taken' as a degree for any structure $\left(X, \leq_{P}\right)$, since $x \leq_{P} y \Rightarrow r \leq$ $r$. Anyway, these functions are only accepted as $\mathfrak{L}$-degrees for the predicates $P_{r}=$ constantly $\mathrm{r}$, in which case $\leq_{P_{r}}=\leq_{P_{r}}^{-1}==_{P_{r}}$, since $x \leq_{P_{r}} y \Leftrightarrow \mu_{P}(x)=\mu_{P}(y) \stackrel{r}{=} r$, for all $x, y$ in $X$.

Constant predicates are not meaningless, since $\leq_{P_{r}}={ }_{P_{r}}=X \times X \neq \emptyset$.

If the poset $\mathfrak{L}$ has a minimum element $\alpha \in L$, and a maximum element $\omega \in L$, then there are the two degrees $\mu_{\alpha}(x)=\alpha, \mu_{\omega}(x)=\omega$, for all $x \in X$, corresponding to the constant predicates $P_{\alpha}=$ constantly $\alpha$, and $P_{\omega}=$ constantly $\omega$, respectively.

\section{L-sets}

Given a triplet $\left(X, \leq_{P}, \leq_{\mu_{p}}\right)$, it is possible to represent the 'collective' that $P$ generates in $X$, translating into mathematical terms the 'collective of the $P$ s in $X$ ' by an L-set (by following [3]), noted by $\mathbb{P}$, and defined by the change of notation:

$x \in_{r} \mathbb{P}$ (read: $x$ belongs to $\mathbb{P}$ with degree $r \in L$ )

$\Leftrightarrow \mu_{P}(x)=r$,

and provided,

$$
\mathbb{P}=\mathbb{Q} \Leftrightarrow \mu_{P}(x)=\mu_{Q}(x), \text { for all } x \in X .
$$

It is obvious that $\mathbb{P}$ is mathematically equivalent to $\mu_{P}$. Hence, the L-set concept is relative to the poset $\mathfrak{L}$ and the chosen $L$-degree, $\mu_{P} \in L^{X}$. There is not, in general, a unique $L$-set in $X$ defined by $P$.

If $P$ is meaningless in $X$, from $\leq_{P}=\emptyset$ it follows that for no $\mathfrak{L}$ a degree does exist: meaningless predicates $P$ do not define any $L$-set $\mathbb{P}$ in the universe of discourse.

It should be distinguished the before mentioned predicate $P_{\alpha}$, with degree $\mu_{\alpha}$, that gives the L-set $\mathbb{P}_{\alpha}$ characterized by $x \in \in_{\alpha} \mathbb{P}_{\alpha}$ for all $x \in X$, and the predicate $P_{\omega}$ whose corresponding L-set $\mathbb{P}_{\omega}$ is characterized by $x \in_{w} X$, for all $x \in X$. For all the constant predicates, the corresponding L-sets $\mathbb{P}_{r}$ are unique, and $\mathbb{P}_{\alpha}, \mathbb{P}_{\omega}$, are their limiting cases.

The definition:

$\mathbb{P} \subset \mathbb{Q}(L$-set $\mathbb{P}$ is included in the $L$-set $\mathbb{Q})$ $\Leftrightarrow \mu_{P}(x) \leq \mu_{Q}(x)$, for all $x \in X$,

obviously gives a reflexive, antisymmetric and transitive relation. That is, $\subset$ is a partial order, under which

$$
\mathbb{P}_{\alpha} \subset \mathbb{P} \subset \mathbb{P}_{\omega}
$$

for all $L-$ set $\mathbb{P}$.

Obviously, it is $\mathbb{P}_{r} \subset \mathbb{P}_{s}$ if and only if $r \leq s$. Consequently, the $L$-set $\mathbb{P}_{\alpha}$ can be identified with the empty set, and the set $\mathbb{P}_{\omega}$ with the total set, that is, with the classical sets $\emptyset$ and $X$, respectively. The set $L^{X}$ can be also taken as that of all 'potential' $L$-sets in $X$.

In the case the poset $\mathfrak{L}$ has the extreme elements $\alpha, \omega$, the set $L_{0}=\{\alpha, \omega\} \subset L$ gives the poset $\mathfrak{L}_{0}=$ $\left(L_{0}, \leq\right)$, and the functions $\mu \in L_{0}^{X}$ are those for which either $\mu(x)=\alpha$, or $\mu(x)=\omega$, for any $x \in X$. The mapping $\varphi: L_{0}^{X} \rightarrow \mathcal{P}(X), \varphi(\mu)=\mu^{-1}(\omega) \subset X$ is bijective, since:

1. $\varphi(\mu)=\varphi(\sigma) \Leftrightarrow \mu^{-1}(\omega)=\sigma^{-1}(\omega) \Leftrightarrow \mu=\sigma$

2. If $A \in \mathcal{P}(X)$, with $\mu_{A}(x)=\left\{\begin{array}{ll}\omega & \text { if } x \in A \\ \alpha & \text { if } x \notin A\end{array}\right.$, is $\mu_{A} \in L_{0}^{X}$ and $\varphi\left(\mu_{A}\right)=A$.

Of course, it is $A=B \Leftrightarrow \mu_{A}=\mu_{B}$, and $A \subset B \Leftrightarrow \mu_{A}(x) \leq \mu_{B}(x)$, for all $x \in X$. It is also $\varphi\left(\mu_{\alpha}\right)=\emptyset$, and $\varphi\left(\mu_{\omega}\right)=X$. Hence, it can be said that in the set $L^{X}$ of the L-sets in $X$, it is included the classical power set $\mathcal{P}(X)$ of $X$.

If $P$ is a predicate on $X$ such that $\mu_{P} \in L_{0}^{X}$, it is said that $P$ is precise, crisp, or rigid. The corresponding (classical) L-sets are obviously unique. In the classical case of crisp sets $(\mathfrak{L}-$ sets in $\{0,1\}^{X}$, or $\left.\{\alpha, \omega\}^{X}\right)$, that the set $\{0,1\}^{X}$ contains all potential classical subsets of $X$, is expressed by the so-called specification axiom ([4]): For each binary predicate $P$, it exists a single subset $\mathbb{P} \subset X$ whose elements are all the $x \in X$ verifying the property denoted by $P$. For non-binary predicates $P$, it should be previously determined which is the more suitable poset $\mathfrak{L}$ in which the degree can vary and, if possible, verifying $\leq_{P}=\leq_{\mu_{P}}$.

After what has been said, it seems to be clarified that a function $\mu \in L^{X}$ only can represent a predicate $P$ on $X$ (namely, a particular use of $P$ on $X)$ provided $\mu$ verifies the intrinsic property of being an $\mathfrak{L}$-degree for $P$. Any function $\mu \in L^{X}$ only 'defines' an $\mathfrak{L}$-set whenever it exists a predicate $P$ such that $\mu=\mu_{P}$.

\section{Family resemblance with L-sets}

In the path towards searching models for Natural Language, it is needed to capture the linguistic relationships between the linguistic terms in the best possible way than possible. The Wittgenstein's concept of family resemblance [12] reflects the family's air between the uses of some words, and in this paper, the definitions previously given in [2], [11] will be enlarged for pairs of L-sets, defined on different universes of discourse. The new definitions allow to approach the concept of 
migration of a predicate as a particular case of family resemblance that is important for the evolution of the use of words. Notice that by the language's own dynamism it is frequent to apply a word to a new universe of discourse once it is first introduced in another universe.

Let $\mu_{P}: X \rightarrow L_{1}$ be a representation of the use of a predicate $P$, or L-set, $\mathbb{P}$, and let $\mu_{Q}: Y \rightarrow L_{2}$ be a representation of a use of the predicate $Q$, or L-set, $\mathbb{Q}$. Both $L$-sets can be easily compared, if the universes $X$ and $Y$ are totally ordered and there exists an isomorphism $f: Y \rightarrow X$, allowing to check, in the universe of discourse $X$, if both predicates show some kind of 'resemblance'.

First of all, let us introduce some instrumental definitions.

\section{Definition 6.1}

- Let $\left(X, \leq_{X}\right)$ and $\left(Y, \leq_{Y}\right)$ be two posets. A nondecreasing function in $A \subset X, f: X \rightarrow Y$, is a mapping verifying that if $x \leq_{X} y$, then $f(x) \leq_{Y} f(y)$, for $x, y \in A$. In addition, if $f$ is onto and one-to-one, it is an isomorphism.

- Let $X$ be a set and $(L, \leq)$ be a poset with a maximum 1. For any mapping $\mu: X \rightarrow L$, it is $S(\mu)=\{x \in X ; \mu(x)=1\}$. If the poset $(L, \leq)$ is with a minimum 0 , it is $Z(\mu)=\{x \in$ $X ; \mu(x)=0\} . S(\mu)$ is the set of prototypes of $\mu$ and $Z(\mu)$ that of the anti-prototypes.

With these concepts, the following definitions can be introduced,

Definition 6.2 Let $X, Y$ be two universes of discourse endowed, respectively, with total orders $\leq_{X}$ and $\leq_{Y}$, and $f: Y \rightarrow X$ an isomorphism. Let $\left(L_{1}, \leq_{L_{1}}\right)$ and $\left(L_{2}, \leq_{L_{2}}\right)$ be two posets with minimum and maximum. $\mu \in L_{1}^{X}$ and $\sigma \in L_{2}^{Y}$ are said to be in the relation of family resemblance, denoted by $(\mu, \sigma) \in \boldsymbol{f r}$, whenever:

1. $Z(\mu) \cap f(Z(\sigma)) \neq \emptyset$, and $S(\mu) \cap f(S(\sigma)) \neq \emptyset$

2. $\sigma$ is non-decreasing in $A \subset Y$ iff $\mu \circ f$ is nondecreasing in $A$.

3. $\sigma$ is decreasing in $A \subset Y$ iff $\mu \circ f$ is decreasing in $A$.

This definition generalizes that given in [11], [2] which only deals with fuzzy sets in the same universe of discourse, and is actually a particular case of Wittgenstein's idea [12]. Notice that with degrees or L-sets, the philosophical problem of the non determination of the concepts extension by resemblance disappears [6].

From this definition follows:

- For no negation $\mu^{\prime}=N \circ \mu$ (see [9]), is $\left(\mu, \mu^{\prime}\right) \in$ fr, because the pair $\left(\mu, \mu^{\prime}\right)$ does not verify the points 2 and 3 in definition 6.2. So, two contradictory fuzzy sets do not verify the relation of family resemblance.
- For no opposite ${ }^{\prime} \mu=\mu \circ \alpha$ (see [9]), is $\left(\mu,{ }^{\prime} \mu\right) \in$ fr. A predicate $P$ represented by $\mu$ and its antonym built as $\mu \circ \alpha$, with $\alpha$ a symmetry (i.e. $\alpha: X \rightarrow X$, such that $\alpha \circ \alpha=i d$ and $\left.\alpha\left(1_{X}\right)=0_{X}\right)$, does not verify the relation of family resemblance since if the pair $\left(\mu,{ }^{\prime} \mu\right)$ verifies properties 2 and 3 of definition 6.2 , then ' $\mu$ introduces the same order in the universe of discourse, and this is contradictory with the concept of antonym (see [9]).

The relation fr of family resemblance is reflexive, $(\mu, \mu) \in \mathbf{f r}$, for all $\mu \in L_{1}^{X}$. It is also symmetric, since $(\mu, \sigma) \in$ fr implies,

1. $Z(\mu) \cap f(Z(\sigma)) \neq \emptyset, S(\mu) \cap f(S(\sigma)) \neq \emptyset$

2. $\sigma$ is non-decreasing in $A \subset Y$ iff $\mu \circ f$ is nondecreasing in $A$.

3. $\sigma$ is decreasing in $A \subset Y$ iff $\mu \circ f$ is decreasing in $A$.

which is equivalent to

1. $f^{-1}(Z(\mu)) \cap Z(\sigma) \neq \emptyset, f^{-1}(S(\mu)) \cap S(\sigma) \neq \emptyset$

2. $\sigma \circ f^{-1}$ is non-decreasing in $A \subset X$ iff $\mu$ is non-decreasing in $A$.

3. $\sigma \circ f^{-1}$ is decreasing in $A \subset X$ iff $\mu$ is decreasing in $A$.

So, $(\sigma, \mu) \in \mathbf{f r}$.

Notwithstanding, fr is not transitive, since there are $\mu, \sigma, \delta$ such that $(\mu, \sigma) \in \mathbf{f r},(\sigma, \delta) \in \mathbf{f r}$, but $(\mu, \delta) \notin \mathbf{f r})$. For example, in the figure 1 , it is $S(\mu) \cap S(\delta) \neq \emptyset$. The lack of transitivity seems in agreement with the idea of people family air translated by fr.

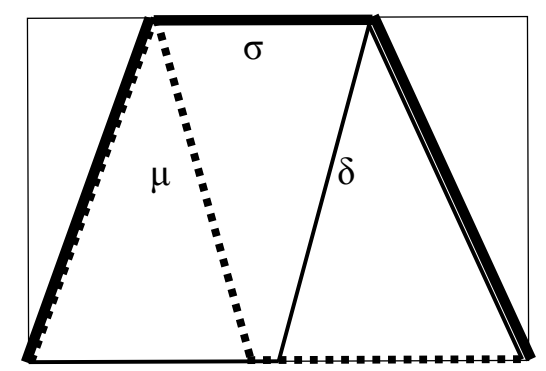

Figure 1: Non transitivity

Remark 6.3 Since monotonicity plays a central role in Definition 6.2, it is not surprising the existence of some link between relation fr and a Galois connection preserving the coincidence of some prototypes and anti-prototypes.

Definition 6.4 (Galois Connection) Given a two posets $(X, \leq)$ and $(Y, \preceq)$, a Galois Connection $G=<X, Y, \alpha, \gamma>$ is a couple of mappings $\alpha: X \longrightarrow Y$ and $\gamma: Y \longrightarrow X$ such that $\alpha(r) \preceq u \Longleftrightarrow r \leq \gamma(u) \quad \forall r \in X, u \in Y$. 
Usually, the mapping $\alpha$ is called the lower adjoint, or coadjoint, while the mapping $\gamma$ is called the upper adjoint, or adjoint.

Since, in the definition of family resemblance, the function $f$, that can be considered the adjoint of the Galois connection, is an isomorphism, the coadjoint is the inverse function.

Therefore, through such Galois connection, from $\mu \in[0,1]^{X}$, it can be built a fuzzy set $\sigma=\mu \circ f$ in family resemblance with $\mu$, because the function $f$ allows to keep the monotonicity, and $Z(\mu) \cap f(Z(\sigma)) \neq \emptyset, S(\mu) \cap f(S(\sigma)) \neq \emptyset)$, since $f(Z(\sigma)) \subset Z(\mu)$ and $f(S(\sigma)) \subset S(\mu)$.

Let, $X$ and $Y$, be endowed with the order induced by a predicate $P$, then $\mu \in[0,1]^{X}$ and $\sigma=\mu \circ f$ (normalized fuzzy sets) are in family resemblance. If $\mu_{P}$ perfectly reflects the meaning of the predicate $P$ in the universe $X$ (i.e. $\leq_{P}=\leq_{\mu_{P}}$ or $x_{1} \leq_{P} x_{2} \Leftrightarrow$ $\left.\mu\left(x_{1}\right) \leq \mu\left(x_{2}\right)\right)$, it can be taken as the representant of the family of fuzzy sets collecting the meaning of $P$. And $\hat{\mu_{P}}$ in the universe $Y$, built through a bijective Galois connection (for instance, the Galois connection obtained taking the adjoint function $f$ in the definition 6.2), $\hat{\mu_{P}}=\mu_{P} \circ f$ can be said that perfectly reflects the meaning of $P$, by the following chain $y_{1} \leq y \Leftrightarrow f\left(y_{1}\right) \leq f(y) \Leftrightarrow \mu \circ f\left(y_{1}\right) \leq \mu \circ f(y)$.

\section{Example 6.5}

1. Fuzzy sets $\mu, \sigma$ in figure 2 verify $(\mu, \sigma) \in \mathbf{f r}$, since $Z(\mu) \cap Z(\sigma)=[0,2] \neq \emptyset$, and $S(\mu) \cap$ $S(\sigma)=\{10\} \neq \emptyset$, and both are non-decreasing in $X=[0,10]$.

2. Fuzzy set $\mu$ in figure 2, and fuzzy set $\lambda$ in figure 3, verify $(\mu, \lambda) \notin \mathbf{f r}$, since $S(\mu) \cap S(\lambda)=\emptyset$, and $\lambda$ is decreasing in $[0,2]$, but $\mu$ is not.

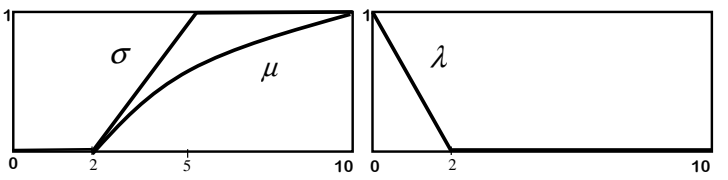

Figure 2:

Figure 3:

3. Sets $[\mu]=\left\{\sigma \in[0,1]^{X} ;(\mu, \sigma) \in \mathbf{f r}\right\}$ are not empty and cover $[0,1]^{X}$, because it is $\bigcup_{\mu \in[0,1]^{X}}[\mu]=[0,1]^{X}$, but they do not give a partition of $[0,1]^{X}$. Anyway, $[\mu]$ can be called the family of $\mu \in[0,1]^{X}$, and each $\sigma \in[\mu]$ a relative of $\mu$, although $\mu$ could have relatives in other families, like it happens in a people family.

4. Fuzzy sets $\mu, \sigma$ in figure 4 verify $\sigma=\mu \circ \alpha$, with the symmetry $\alpha(x)=10-x$, represent two antonyms. Obviously, $\mu$ and $\mu \circ \alpha$ do not show family resemblance.

5. Fuzzy sets $\mu, \sigma$ in figure 5 verify $\sigma=\mu \circ u^{-1}$, with $u(x)=\sqrt{x}$, and show family resemblance.
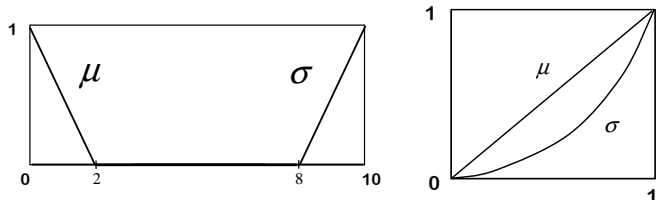

Figure 4:

Figure 5:

\section{Predicates Migration}

Definition 7.1 Suppose that

- $X$ and $Y$ are two universes of discourse endowed with total orders $\leq_{X}$ and $\leq_{Y}$ respectively and that it exists an order isomorphism $f: Y \rightarrow X$ between $\left(Y, \leq_{Y}\right)$ and $\left(X, \leq_{X}\right)$.

- $\left(L_{i}, \leq_{i}\right)$ are two posets with maximums $1_{i}$, and minimums $0_{i}$, respectively, and that it exists a non-decreasing function $F: L_{1} \rightarrow L_{2}$ (i.e. $a \leq$ $b \Rightarrow F(a) \leq F(b))$, such that $F\left(0_{1}\right)=0_{2}$, and $F\left(1_{1}\right)=1_{2}$.

If two fuzzy sets $\mu \in L_{1}^{X}$, and $\sigma \in L_{2}^{X}$, verify

1. $f(Z(\sigma)) \cap Z(\mu) \neq \emptyset, f(S(\sigma)) \cap S(\mu) \neq \emptyset$.

2. $\sigma=F \circ \mu \circ f$,

it is said that $\sigma$ is a migration of $\mu$.

It is immediate that if $\sigma$ is a migration of $\mu$, then $(\mu, \sigma) \in \mathrm{fr}$, although obviously the reciprocal is not true. Provided $\sigma=\mu_{Q}$ and $\mu=\mu_{P}$ it can be said that $Q$ is a migration of $P$.

Theorem 7.2 Provided $\mu \in[0,1]^{X}, \sigma \in[0,1]^{Y}$, with $X, Y$ contained in $\mathbb{R}$, and there are

- A non-decreasing bijective function $f: Y \rightarrow$ $X$, such that $f(Z(\sigma)) \subset Z(\mu)$, and $f(S(\sigma)) \subset$ $S(\mu)$.

- A non-decreasing function $F:[0,1] \rightarrow[0,1]$, such that $F(0)=0$, and $F(1)=1$.

if $\sigma=F \circ \mu \circ f$, then $\sigma$ is a migration of $\mu$.

Proof. Immediate.

Theorem 7.3 All migration $\sigma \in[0,1]^{Y}$ from $\mu \in$ $[0,1]^{X}$, keep a family resemblance with $\mu$.

Proof. Immediate.

Example 7.4 Let $P$ be the predicate 'short' defined in $[0,200]$ by $\mu_{s}(x)=1-\frac{x}{200}$. Let $f:[0,1] \rightarrow$ $[0,200], f(x)=200 \cdot x$, and $F=I d$ :

$$
\left(F \circ \mu_{s} \circ f\right)(y)=\mu_{s}(f(y))=\mu_{s}(200 y)=1-y
$$

that represents $Q=$ tall.

With $F(x)=x^{2}$, it is $\left(F \circ \mu_{s} \circ f\right)(y)=(1-$ $y)^{2}=y^{2}-2 y+1$, reflecting another use of 'small' in $[0,1]$. Consequently, it can be said that 'small' is a migration to $[0,1]$ of 'short' in [0,200]. 


\section{Remark 7.5}

- A possible definition of when two predicates $P$ and $Q$ in a universe of discourse $X \subset R$ are exact synonyms could be done by the equality, $\mu_{Q}=\mu_{P} \circ f$, once they are represented by fuzzy sets $\mu_{P}, \mu_{Q}$ in $[0,1]^{X}$, and $f: X \rightarrow X$ is a bijective function such that:

$$
\begin{aligned}
& \text { 1. } x \leq y \rightarrow f(x) \leq f(y) \\
& \text { 2. } f\left(Z\left(\mu_{Q}\right)\right) \subset Z\left(\mu_{P}\right) \\
& \text { 3. } f\left(S\left(\mu_{Q}\right)\right) \subset S\left(\mu_{P}\right)
\end{aligned}
$$

Notice that this definition is a particular case $(F=i d)$ of definition 7.1. Is in this sense that an exact synonym can be viewed as a migration. Then, predicates in example 7.4 can be taken as exact synonyms.

- Although definition 6.2 is only for a totally ordered universes, it is not a too limiting one since, in most practical cases (like that in 3.2, point 2), the elemental meaning comes from some equivalence in $\mathbb{R}$.

\section{Conclusions}

In his Philosophical Investigations, Wittgenstein conceived language in a way close to how people manages it. Thus, the meaning of an imprecise predicate is not given by necessary and sufficient conditions, but is built up by some similarity, that partially preserves prototypes and is done in processes that evolve over tie and finish when the predicate becomes obsolete. Notwithstanding, for more complex predicates like $P=$ beautiful in a set of art objects, it could be not clear enough the existence of prototypes and, since in such cases it could be $S\left(\mu_{P}\right)=\emptyset$, the study of the family resemblance for these predicates remains an open problem.

It should be pointed out that the definition of family resemblance introduced in this paper is essentially done through monotonic functions between the (totally ordered) universes of discourse. It is for this reason that it seems suitable to consider its relation with some Galois Connection.

In natural language, families $[\mu]$ of 'relatives' must be 'open', but not 'closed' like they were defined. These families are here static (sets), but in natural language they should have a dynamic character. With time, an element $\sigma$ that was not in $[\mu]$, could be included in $[\mu]$ and thus generating a new family, indeed, changing the relation fr. Actually, fr is not a permanent relation in natural language, it is a changing one. Only with families of resemblance taken as classical sets, human thought seems to be impossible (see [5]). It is for reasons like this, that this paper cannot be seen as anything else than some unended initial reflections on the representation of the concepts of family resemblance and predicates linguistic migration. It yet lacks, for instance, a study of family resemblance in non-totally ordered universes.

\section{References}

[1] See any Google's entrance on 'Montessori Methods' For instance, http://en.wikipedia.org/wiki/Montessori_method.

[2] I. García-Honrado, E. Trillas, and S. Guadarrama. Grado de parentesco entre predicados. In Actas XV Congreso Español sobre Tecnologías y Lógica Fuzzy, pages 163-168, 2010. (In Spanish).

[3] J. Goguen. L-fuzzy sets. Jour. Math. Anal. Appl., 10:145-174, 1967.

[4] P. R. Halmos. Naïve set theory. Van Nostrand, New York, 1960.

[5] S. Pinker. Words and Rules. Basic Books, N.Y., 1999.

[6] B. Prien. Family resemblance. A Thesis about the Change of meaning over time. Kriterion, (18):15-24, 2004.

[7] E. Trillas. On the use of words and fuzzy sets. Information Sciences, 176(11):1463-1487, 2006.

[8] E. Trillas. On the genesis of fuzzy sets. Agora, 27(1):7-33, 2008.

[9] E. Trillas. On a model for the meaning of predicates. In Rudolf Seising, editor, Fuzzy sets theory. Philosohpy and criticism, volume 243 of Studies in Fuzziness and Soft Computing, chapter 9, pages 175-205. Springer Verlag, 2009.

[10] E. Trillas and C. Alsina. A reflection on what is a membership function. Mathware 85 SoftComputing, 6:201-215, 1999.

[11] E. Trillas, C. Moraga, and A. Sobrino. On 'family resemblances' with fuzzy sets. In Proceedings IFSA-EUSFLAT, pages 306-311, 2009.

[12] L. Wittgenstein. Philosophical Investigations. Basil Blackwell, Oxford, 3rd edition, 1981.

[13] L. Wittgenstein. Tractatus logicophilosophicus. Routledge, 2001.

[14] L. A. Zadeh. Fuzzy Sets. Information and Control, 8:338-353, 1965.

[15] L. A. Zadeh. From computing with numbers to computing with words.from manipulation of measurements to manipulation of perceptions. In P.P. Wang, editor, Computing with words, pages 35-68. John Wiley \& Sons, 2001. 